\title{
Diabetic Ketoacidosis due to Simultaneous Acute-onset Type 1 Diabetes and Graves’ Disease
}

Chikaaki Kobashi', Emiri Muranaka1, Kanako Motoyoshi', Yukihiro Satou', Tadashi Ikeda ${ }^{2}$, Kouichi Shigeta ${ }^{1}$ and Masaharu Urakaze ${ }^{1 *}$

${ }^{1}$ Kamiichi General Hospital, Medicine and Diabetic Center, Kamiichi-machi, Toyama, Japan

2Ikeda Clinic, Kamiichi-machi, Toyama, Japan

\begin{abstract}
An 18-year old Japanese woman with thirst, weight loss and palpitations for a few weeks' duration was admitted. Her diagnosis was diabetic ketoacidosis with acute-onset type 1 diabetes (T1D) and Graves' disease (GD) (autoimmune polyglandular syndrome type 3 variant; APS3v). She was treated with intensification therapy with insulin and anti-thyroid drugs. Human leukocyte antigen testing revealed that her HLA type was HLA- DRB1*08:02DQA1*03:01-DQB1*03:02 haplotype, which was vulnerable not only to APS3v, but also to both acute-onset T1D and GD. This genetic factor probably contributes to the simultaneous occurrence of T1D and GD.
\end{abstract}

Keywords: Diabetic ketoacidosis; Type 1 diabetes; Graves' disease; Autoimmune polyglandular syndrome; Human leukocyte antigen

\section{Background}

Diabetic ketoacidosis (DKA) is a serious complication for diabetic patients. Fairly infrequently, thyroid storm induces DKA. The thyroid storm which is in a state which occurs from the failure of the compensation mechanism of the body for the excessive thyroid hormone activity is induced by the some factors in the patients with thyrotoxicosis. Here, we report a rare case in which DKA and thyrotoxicosis occur approximately at the same time.

If you deal with cases of DKA or thyroid crisis, it seems essential to be mindful of the possibility of the coexistence of these two diseases.

\section{Introduction}

Diabetic ketoacidosis (DKA) is a serious metabolic disorder due to depletion of insulin activity. Although persons with DKA typically have a history of diabetes, around $30 \%$ have newly diagnosed diabetes, mainly type 1 diabetes mellitus. When this pathological state traces the serious course due to inappropriate diagnosis and deficiency of the treatment, the patients may return to the poor outcome, with the death rate reported to be less than 2\% [1]. Another endocrinal critical state is thyroid storm, is a state of excessive thyroid hormone activity, mainly due to Graves' disease [2]. In a rare case that both DKA and thyroid storm occur simultaneously, clinical feature of thyroid storm is masked by DKA [3]. We herein report a case of diabetic ketoacidosis due to acute-onset type 1 diabetes mellitus with simultaneously coexistent Graves' disease.

\section{Case}

An 18-year-old Japanese woman was hospitalized with a four-week history of thirst, palpitations, general malaise and a fourteen kilogram weight loss. Her past medical history was unremarkable, but her mother had Graves' disease. On admission, she was $154 \mathrm{~cm}$ in height and weighed $40.6 \mathrm{~kg}$ (body mass index: $17.1 \mathrm{~kg} / \mathrm{m}^{2}$ ). Her blood pressure was $154 / 104 \mathrm{mmHg}$, her pulse was regular at 141 beats/min and her temperature was $36.9^{\circ} \mathrm{C}$. Exophthalmos was not observed. A mild diffuse goiter was palpable without tenderness. The skin was slightly dry, and turgor was decreased. There were no abnormal findings of the heart, lungs or abdomen, except tachycardia. Neurological examinations revealed no abnormalities. Ophthalmological examinations revealed no signs of diabetic retinopathy. The plasma glucose and HbAlc levels were markedly elevated. The serum and urinary ketone bodies also increased. Arterial blood gas data showed a metabolic acidosis, with an increased anion gap. The serum and urinary C-peptide levels were remarkably decreased. She was positive for anti-glutamic acid decarboxylase (GAD) and IA-2 antibodies. On the basis of the diagnosis of DKA, the patient was treated with a physiological saline infusion for correction of dehydration and insulin infusion therapy for correction of hyperglycemia. Although dehydration and hyperglycemia were promptly remedied, tachycardia remained persistent. Because tachycardia was not able to be explained alone by DKA-related dehydration, the thyroid disease was added to the differential diagnosis. The level of FT3 was within normal range, but the level of FT4 was slightly elevated and the level of TSH was below the detectable limit. She was positive not only for TSH-binding inhibitor immunoglobulin (TBII) and thyroid-stimulating antibodies (TSAb), but also for antithyroid peroxidase antibodies (TPOAb) and anti-thyroglobulin antibodies (TgAb). Ultrasonography of thyroid revealed a diffusely swollen thyroid gland with both heterogeneously low echogenicity and the increase in the blood flow. The chest X-ray findings were normal. Electrocardiography revealed sinus tachycardia. Based on the above results, the patient was diagnosed to have diabetic ketoacidosis with acute onset T1D and Graves' disease (autoimmune polyglandular syndrome type 3 variant, APS3v). After intravenous infusions of fluids and insulin, intensive insulin therapy (maximum total $1.1 \mathrm{units} / \mathrm{kg} /$ day) was initiated. The diabetic ketoacidosis disappeared within one day and her blood glucose control was improved. Treatment with thiamazole ( $15 \mathrm{mg} /$ day) relieved thyrotoxicosis. Two months later, thyroid hormone had been normalized. The patients are still cared for in oral thiamazole therapy. In terms of diabetes, at first, this case

*Corresponding author: Masaharu Urakaze, Kamiichi General Hospital, Medicine and Diabetic Center, Kamiichi-machi, Toyama, Japan, Tel: 81764721212; E-mail: murakaze@kamiichi-hosp.jp

Received May 17, 2016; Accepted May 23, 2016; Published May 28, 2016

Citation: Kobashi C, Muranaka E, Motoyoshi K, Satou Y, Ikeda T, et al. (2016) Diabetic Ketoacidosis due to Simultaneous Acute-onset Type 1 Diabetes and Graves' Disease. J Diabetes Metab 7: 671. doi:10.4172/2155-6156.1000671

Copyright: (c) 2016 Kobashi C, et al. This is an open-access article distributed under the terms of the Creative Commons Attribution License, which permits unrestricted use, distribution, and reproduction in any medium, provided the original author and source are credited. 
required maximal insulin aspart dose of 52 unit /day. Blood glucose control was accomplished in insulin glargine (16 units) and insulin aspart (14 units). Currently, four years after her first visit, she continues intensive insulin therapy (total 0.7 units/kg/day) for her T1D, and has remained euthyroid with anti-thyroid drugs, to date. The patient's HLA typing results were as follows: HLA- DRB1*08:02-DQA $1^{\star} 03: 01$ DQB1*03:02 haplotype.

\section{Discussion}

Thyroid storm and DKA are both fatal potentially and the prognosis depends on whether or not these conditions are recognized and treated adequately [4]. Our current case covered thyrotoxicosis with Graves' disease by DKA. She lacked characteristic sign of thyrotoxicosis. She was diagnosed as presenting DKA, and was treated promptly. It was reported that discovery of thyrotoxicosis tended to be late in the DKA patients for suppression of fever [1,3], relatively low thyroid hormone levels in tune with acute increased stress [4]. Because a symptom of DKA was similar to that of thyrotoxicosis, the immediate diagnosis of thyrotoxicosis was difficult. However, in this case, presence of thyrotoxicosis were suspected in a relatively initial stage based on tachycardia because a symptom of Graves' disease was not observed before the onset, and that it was not relieved in spite of revision of the dehydration associated with DKA. Thus, the treatment with betaadrenergic blocking drug and thiamazole was started relatively quickly and the patient is good as for this early treatment.

It was not certain that Graves' disease was present 4 weeks prior to administration. With regard to the influence of Graves' disease on diabetes mellitus, the deterioration of glucose intolerance under a hyperthyroid state was observed [4]. It was reported that Graves' disease increased the intestinal absorption of carbohydrates and productions of liver glucose from glycogen for liver insulin resistance. It is known that the glucose uptake of the skeletal muscle is lower in hyperthyroid patients than in euthyroid patients. The factors worsened abnormal glucose metabolism and insulin deficiency, which leads to the onset of DKA in this case.

Whereas, thyroid storms are usually induced by infection, trauma and a surgical emergency or surgery more generally by isotope radiation of thyroid gland, DKA, toxemia of pregnancy or the delivery [5]. Therefore, DKA cannot be ignored as a factor inducing the onset of the thyroid storm by thyrotoxicosis [5]. DKA and thyroid crisis are fatal potentially and the prognosis is depending on treatment in earliness. Therefore, it seems to be important that we keep likelihood of the coexistence of these two diseases in a heart when we deal with a case of DKA. This case report is presented by this perspective.

Type 1 diabetes mellitus (T1D) and autoimmune thyroid disease (AITD) are common autoimmune endocrine diseases, and the occurrence of both T1D and AITD in the same patient is defined as APS3v. Epidemiological evidence suggests that Graves' disease was reported to be present in $6.3 \%$ of patients with T1D (acute-onset T1D, $2.9 \%$, and slowly progressive T1D, $13.0 \%$ ) and $0.4 \%$ with type 2 diabetes mellitus (T2D) in a Japanese outpatient clinic [6]. However, the simultaneous occurrence of acute-onset T1D and Graves' disease is relatively rare. With regard to the clinical characteristics of APS3v, marked female predominance, a slowly progressive clinical course with older T1D onset age, a higher prevalence of GAD antibodies and a higher level of GAD antibodies were observed in APS3v patients compared to T1D patients without AITD [7]. The prevalence of IA-2 antibodies was significantly higher in acute-onset T1D than in slowly progressive T1D among Japanese APS3v patients [7]. The HLA types of the present patient was $\mathrm{DRB} 1^{*} 08$ :02-DQA1 ${ }^{\star} 03: 01-\mathrm{DQB1} 1^{\star} 03: 02$ haplotype. For HLA class II and APS3v, HLA-DRB $1^{\star} 04: 05-D Q B 1{ }^{*} 04: 01$, DRB $1^{\star} 09: 01-$ DQB $1^{\star} 03: 03$ and $\mathrm{DRB} 1^{\star} 08: 02-\mathrm{DQB} 1^{\star} 03: 02$ are considered to be the three major susceptibility HLA haplotypes for T1D in the Japanese population. Hashimoto et al. [8] reported that the characteristics of susceptibility HLA haplotypes differed among patients with APS3v (T1D and Graves' disease), T1D alone and Graves' disease alone. The haplotype frequencies of HLA DRB1 ${ }^{\star} 04$ :05-DQA ${ }^{\star} 03$ :03-DQB ${ }^{\star} 04: 01$ and $\mathrm{DRB} 1^{\star} 08: 02-\mathrm{DQA} 1^{\star} 03: 01-\mathrm{DQB} 1{ }^{\star} 03: 02$ were significantly higher in APS3 patients with both T1D and Graves' disease than in controls. With regard to the pathogenic mechanisms that contribute to the development of APS3v, there are several hypothetical mechanisms by which HLA could be involved in a common etiology of T1D and AITD [9]. First, some HLA susceptibility alleles for T1D might be in tight linkage disequilibrium with that for AITD. Second, the HLA class II molecules might share a similar HLA pocket structure, fitting both islet cell peptides and thyroidal peptides. On the other hand, GAD might contribute to the association between T1D and Graves' disease, because of the presence of GAD activity in the thyroid and the relationship not only between GAD antibody and T1D, but also between GAD antibody and Graves' disease. A high-titer of GAD antibody is associated with the development of AITD in patients with T1D. Conversely, AITD patients (particularly Graves' disease patients) with a high titer of GAD antibody and/or who were positive for both GAD and IA-2 antibodies are prone to develop T1D [10]. In the present patient, T1D and Graves' disease apparently developed almost simultaneously, which is relatively rare in Japan.

In conclusion, we have described a Japanese woman with APS3v, in whom acute-onset T1D and Graves' disease developed almost simultaneously. She was found to be genetically susceptible to not only APS3v (T1D and Graves' disease), but also to both acute-onset T1D and Graves' disease. The genetic factor probably make a contribution to the simultaneous occurrence of acute-onset T1D and Graves' disease in patients with APS3v.

\section{References}

1. Kitabchi AE, Umpierrez GE, Murphy MB (2004) In: International textbook of diabetes mellitus UK: John Wiley, Sons, Ltd; Diabetes ketoacidosis and hyperglycemic hyperosmolar state 21: 1101-1119.

2. Burch HB, Wartofsky L (1993) Life-threatening thyrotoxicosis. Thyroid storm Endocrinol Metab Clin North Am 22: 263-277.

3. Kunishige M, Sekimoto E, Komatsu M, Bando Y (2001) Thyrotoxicosis masked by diabetic ketoacidosis. Diabetes care 24: 171 .

4. Mouradian M, Abourizk N (1983) Diabetes mellitus and thyroid disease. Diabetes care 6: 512-520.

5. Kearney T, Dang C (2007) Diabetic and endocrine emergencies. Postgrad Med J 83: $79-86$.

6. Osaki Y, Kawai K, Motohashi S, Sone H, Yamada N (2009) Type 1 diabetes mellitus and autoimmune thyroid disease in Japanese: prevalence and pattern of onset. J Jpn Diabetes Soc 52: 887-893.

7. Horie I, Kawasaki E, Ando T (2012) Clinical and genetic characteristics of autoimmune polyglandular syndrome type 3 variant in the Japanese population. $\mathrm{J}$ Clin Endocrinol Metab 97: E1043-E1050.

8. Hashimoto $\mathrm{K}$, Maruyama $\mathrm{H}$, Nishiyama M (2005) Susceptibility alleles and haplotypes of human leukocyte antigen DRB1, DQA1, and DQB1 in autoimmune polyglandular syndrome type III in Japanese population. Horm Res 64: 253-260.

9. Huber A, Menconi F, Corathers S, Jacobson EM, Tomer Y (2008) Joint genetic susceptibility to type 1 diabetes and autoimmune thyroiditis: from epidemiology to mechanisms. Endocr Rev 29: 697-725.

10. Moriguchi M, Noso S, Kawabata $Y$ (2011) Clinical and genetic characteristics of patients with autoimmune thyroid disease with anti-islet autoimmunity. Metabolism 60: 761-766. 